\title{
Design, Construction and Programming of a Mobile Robot Controlled by Artificial Vision
}

\author{
Claudio Urrea* and Alex Yau \\ Grupo De Automática, Departamento De Ingeniería Eléctrica, Universidad De Santiago De Chile, Chile
}

Received 10 July 2016; Accepted 10 September 2016

\begin{abstract}
The design, construction and programming of a mobile robot controlled by means of artificial vision, capable of recognizing, grabbing and moving specific objects in a completely autonomous way is presented, together with the conceptual and theoretical-practical grounds for the work. Having a mobile robot like this will allow interesting developments to be made in various areas of mobile robotics.
\end{abstract}

Keywords: Mobile Robot, Design, Construction, Programming.

\section{Introduction}

In recent years there has been increasing interest in research on mobile robots due to the endless number of remote applications that can be developed with them, particularly in areas of high risk to human beings. Currently, thanks to the degree of development that has been reached in the field of mechanisms and sensors, the implementation of systems with a high degree of interaction with the environment has been achieved, allowing robots to get a more accurate description of their environment, such as sampling, analysis of the surrounding, detection of gases, leakage, or even sending video signals so that the observer is not exposed. Since mobile robots can now carry out their tasks with greater accuracy, precision and autonomy, the developments of control methods, as well as the implementation of new prototypes, are of high importance in the field of robotics.

Mobile robots have the advantage of integrating the functionality of a device or system with the mobility of an autonomous vehicle, bringing about a number of advantages, but also a number of yet unsolved technological barriers such as:

- Increased duration of battery charge to achieve greater autonomy.

- Increased potentialities of the navigation systems to allow automatic mobility in the most efficient, flexible, fault-tolerant and safe possible manner.

- Improved efficiency of the control of fleets consisting of various mobile robots to solve problems such as optimized scaling, routing or traffic management, etc.

\section{General Aspects of Robotics}

\subsection{Background of mobile robots}

*E-mail address: claudio.urrea@usach.cl

ISSN: 1791-2377 @ 2016 Eastern Macedonia and Thrace Institute of Technology. All rights reserved.
The use of mobile robots is justified in applications in which tasks that are unpleasant or risky to humans are carried out, among which transporting hazardous materials, mining excavations, industrial cleaning, or inspection of nuclear power plants are examples of situations in which a mobile robot can do the job and avoid uncalled for risks to the workers' health. Another group of applications in which these kinds of robots complement the operator's activities are supervision, inspection, or assisting disabled people. Also in surgical teleoperation applications, where there is considerable backwardness in communications, the use of vehicles with some degree of autonomy is interesting.

An autonomous mobile robot is characterized by having an intelligent connection between the operations of perception and action, which defines its behavior and allows it to fulfill the programmed objectives in surroundings with some uncertainty. The degree of autonomy depends to a large extent on the ability of a robot to disregard the surroundings and convert the obtained information into orders, so that when they are applied to the locomotion system's actuators, the efficient performance of the task is guaranteed. From this it can be concluded that the main characteristics of a mobile robot, in contrast with any other kind of vehicle, are the following:

- Perception, which determines the relation of the robot with its work environment, by means of its onboard sensors.

- Reasoning, which determines the actions that must be carried out at all times according to the state of the robot and its surroundings, to reach the assigned goals.

In this way, the reasoning ability of an autonomous mobile robot is translated into the planning of safe trajectories that will allow it to fulfill the assigned objectives. The execution of a task in particular must be carried out in a closed loop for the robot to adapt to navigating through unstructured surroundings. A traditional 
control loop is not used because the action is not generated by simple output feedback.

It is therefore necessary to use a planner skilled in geometric analysis that knows the conditions of the surroundings and of the mobile robot, together with its kinematic and dynamic characteristics. Starting from this the mobile robot can make the transformation of the data, supplied by the perception, into adequate control references that do not go beyond any of its physical limitations, and that define trajectories free from obstacles that guarantee the achievement of the goals established for the specific task. Thus, the planner becomes responsible, to a large extent, of the mobile robot's navigation efficiency, so its design requires special care. To carry out these tasks it is necessary to have fine control of the movements of the mobile robot, among which the following techniques stand out:

- Pure Pursue.

- PI-PD-PID control techniques.

- Ziegler-Nichols controllers.

- Cohen and Coon methods.

- Fuzzy control.

In addition to the above control techniques, there are methods for the execution of its specific tasks, e.g., the geometric method for following routes explicitly, the methods based on control theory applied to following trajectories and routes, as well as predictive and reactive control following, based on the direct reaction to environmental perception sensors [1,2].

\subsection{Morphology of mobile robots}

The design of the mobile robot that will be constructed is based on a configuration that consists of four wheels. Various configurations that comply with these characteristics are presented below [3-5].

\subsubsection{Ackermann}

The arrangement and mobility of its axles is similar to that of a conventional vehicle. The inner front wheel turns at a slightly larger angle than the outer wheel to prevent sliding. The extensions of the front axles intersect at some point on the extension of the axle of the rear wheels. The locus of the points drawn on the ground by the centers of the wheels are concentric circumferences centered on axle P1, as shown in Figure 1.

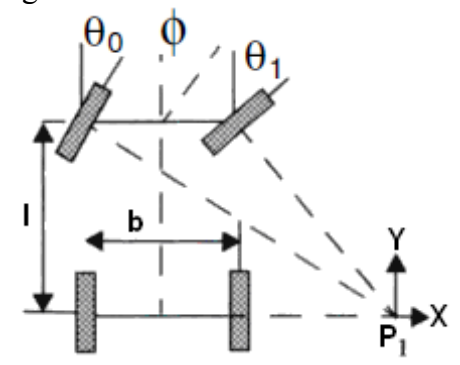

Fig. 1. Ackermann configuration.

\subsubsection{Skid-Steer}

They are composed of several wheels on both sides of the vehicle that act simultaneously. The motion consists of the combination of the movements of the left and right sides, which result in advance, reverse, turning motions, etc. It is important to point out that in contrast with the Ackermann, there is no axle movement.
Other kinds of four-wheel robots exist with hybrid configurations of wheels with differential traction and omnidirectional, as well as others with four omnidirectional wheels. For the design of the mobile robot presented in this paper this four-wheel configuration without axle movement was chosen, thanks to its great maneuverability.

\subsection{Kinematics of mobile robots}

\subsubsection{Representation of the Robot's position}

In the kinematic analysis of the mobile robot, it is considered as a rigid body on four wheels operating on a horizontal plane. To specify the position and orientation of this mobile robot, a two-dimensional movement is established between a global reference framework of the $\left(X_{g}, Y_{g}, Z_{g}\right)$ plane that is chosen arbitrarily, and a local reference framework of the robot $\left(\mathrm{x}_{1}, \mathrm{y}_{1}, \mathrm{z}_{1}\right)$, associated with a point on the frame that corresponds to the robot's Center of Mass (COM). According to Figure 2a, this COM can be described in the global reference system as $\mathrm{COM}=(\mathrm{X}, \mathrm{Y})$, and the angular difference between the global and local system of reference is $\theta$. Therefore, its position and orientation are determined by (1):

$$
\xi_{G}=\left[\begin{array}{l}
X \\
Y \\
\theta
\end{array}\right]
$$

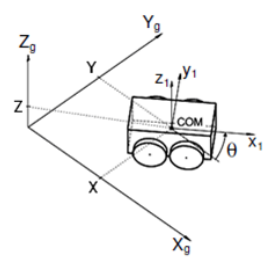

(a)

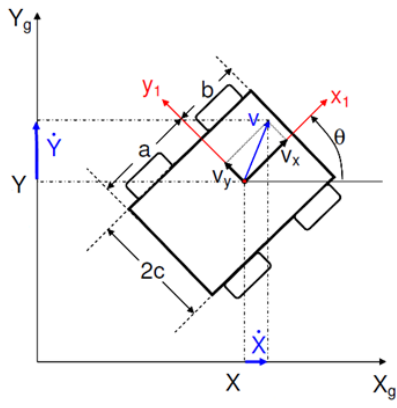

(b)
Fig. 2. a) Reference system. b) Velocities of the robot and global reference.

The velocity vector is defined on a two-dimensional coordinate system of the COM and expressed as a function of the local reference system by means of $V_{x}$ and $V_{y}$, which determine the vehicle's longitudinal and lateral velocities (Figure 2b). To get the robot's movement in global terms, its local movement is mapped along the global reference axes, leading to Equation (2).

$$
\dot{\mathrm{q}}=\left[\begin{array}{c}
\dot{X} \\
\dot{Y} \\
\dot{\theta}
\end{array}\right]=\left[\begin{array}{ccc}
\cos \theta & -\sin \theta & 0 \\
\sin \theta & \cos \theta & 0 \\
0 & 0 & 1
\end{array}\right]\left[\begin{array}{c}
\mathrm{v}_{\mathrm{x}} \\
\mathrm{v}_{\mathrm{y}} \\
\omega
\end{array}\right]
$$

\subsection{Velocity ratio}

The representation of the mobile robot in space does not impose any restriction on its motion in the plane, so only its free body kinematics is described. However, it is necessary to include the ratio between the velocities of the wheels and the local velocities. Then we have that the cinematic model 
for this mobile robot is constituted by the system of equations:

$$
\begin{aligned}
& \dot{X}=r\left(\frac{\omega_{L}+\omega_{R}}{2}\right) \cos \theta+x_{I C R} r\left(\frac{-\omega_{L}+\omega_{R}}{2 c}\right) \operatorname{sen} \theta \\
& \dot{Y}=r\left(\frac{\omega_{L}+\omega_{R}}{2}\right) \operatorname{sen} \theta-x_{I C R} r\left(\frac{-\omega_{L}+\omega_{R}}{2 c}\right) \cos \theta \\
& \dot{\theta}=r\left(\frac{-\omega_{L}+\omega_{R}}{2 c}\right)
\end{aligned}
$$

where $\mathrm{X}_{\mathrm{ICR}}$ is a coordinate of the center of instantaneous rotation, and $\omega_{\mathrm{L}}$ and $\omega_{\mathrm{R}}$ denote the angular velocities of the left and right wheels, respectively. At the kinematic level, they can be considered as control entries and can be used to control the longitudinal and angular velocity according to Equation (6),

$$
\omega=r \frac{-\omega_{L}+\omega_{R}}{2 c}
$$

where $r$ is the presumed effective radius of the wheels and $2 \mathrm{c}$ is the spacing between the wheels. In this way kinematic control of the mobile robot is achieved, as shown schematically in Figure 3.

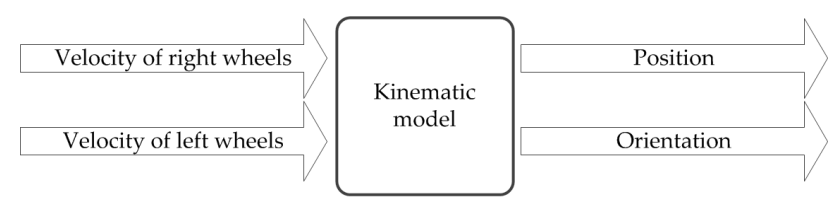

Fig. 3. Kinematic control of the robot.

\section{Guidance of Mobile Robots}

The navigation problem of a mobile robot can be summarized in three questions: Where am I? where am I going?, and how should I get there?, so a navigation system consists in the set of sensors, systems, methods and technologies that try to situate a mobile robot in its surroundings. Now, for a mobile robot to be completely autonomous and be able to go over its work space without the occurrence of any inconveniences, it is necessary to answer the above questions by means of the following stages in the process of mobile robot guidance: localization, mapping and planning of trajectories.

The localization process consists in finding the location of the mobile robot within its work setting. The mapping process consists in creating a representation of the setting in which the mobile robot is performing, which must be comprehensible by it. The trajectory planning consists basically in determining the route that the mobile robot must follow, from an initial to a final configuration. This trajectory must be planned in such a way that the mobile robot does not collide with objects that it finds along its way.

\subsection{Localization}

The concept of localization arises as a need to estimate the position of the mobile robot within the work setting (map). There are different localization techniques that can be grouped into two kinds:
- Local Localization, which consists in determining the position of the mobile robot from a known initial position; i.e., follow-up the movements made by the mobile robot, estimate its degree of advance and orientation, and make calculations to estimate the current position from the starting point of the movement, for example, odometry and landmarks may be mentioned.

- Global Localization, which consists in finding the position of the mobile robot without knowing its location at a previous instant, or having uncertain information. Kalman filtering, Markov filtering, and particle filtering may be mentioned.

\subsection{Map generation}

It is important for the mobile robot to get in some way a map of the surroundings in which it must move. This must be done so that in a later stage it can use that map to trace the trajectory that it must follow.

\subsubsection{Sensory maps}

They are based on invariant characteristics or on grid maps of spatial occupation (probabilistic). In the grid maps the space is represented as a set of $2 \mathrm{D}$ or $3 \mathrm{D}$ cells, associating an occupation (1) or free (0) state. The number of cells is $\left(n^{d}\right)$, where $n$ is the number of cells per dimension and $d$ is the number of dimensions.

\subsubsection{Geometric maps}

It is considered that the space can be represented by a series of geometric primitives (such as straight lines or polygons). The exploration is based on algorithms inspired in computational geometry.

\subsubsection{Topological maps}

A map of the different situations that the robot will face is constructed. Then this map is represented based on a graph, so when the map indicates a change of situation, if it does not exist any longer, a new node and an arc are included. The resultant graph represents a map of the free space of the surroundings.

\subsection{Trajectory planning}

The trajectory planning problem consists in determining a trajectory between an initial and a final configuration, so that the mobile robot does not collide with the obstacles and complies with its kinematic restrictions. To do this it is necessary to consider the following specifications: minimum distance trajectory, semi-unknown environments, dynamic environments, additional restrictions, and efficiency.

To tackle the trajectory planning problem there are various methods that are grouped into graph search, dynamic programming, Voronoi diagrams, and visibility graphs.

\section{Choice of Electric Components}

The electric components chosen for the designed and implemented mobile robot are presented below:

- Servomotors. The total number of de servomotors of the mobile robot is 17 .

- Microcontroller. The microcontroller used is a Basic Stamp by Parallax.

- Servo controller. Because of the large number of servomotors used in the configuration of the mobile 
robot and for a better compatibility performance, the Parallax Servo Controller (PSC) board is used.

- Sensors. Due to the various tasks that this mobile robot will carry out, two types of sensors are chosen: an ultrasonic distance sensor, and a shape and color sensor (Acroname CMUCam 2+).

- Batteries. Two 6-V rechargeable batteries are used.

- Computer. To improve the processing capacity with respect to the artificial vision task, a netbook was incorporated.

\section{Construction of the Mobile Robot}

The construction process of this mobile robot was divided into three main mechanical stages: the frame of the mobile robot, a manipulator with clamp as terminal effector, and a manipulator of the artificial vision camera.

\subsection{Frame of the mobile robot}

The frame of the mobile robot is the structure that integrates all its components, i.e., the camera with its manipulator, the manipulator, batteries, circuits, and sensors, among other components, forming the robot's main structure. The design criteria were selected considering that the mobile robot has the following characteristics:

- Great mobility.

- Acceptable autonomy time.

- Light and easily transportable.

- Expansion and connectivity capacity.

- Acceptable construction cost.

A rail capable of displacing the artificial vision camera linearly to increase its range of vision. This configuration was performed imitating the motion of the head of a turtle, in this way increasing its vision range by almost $180^{\circ}$.

Therefore, in its final design, the mobile robot consists of the following elements:

- Base0. Inside there are two supports for each wall for the servomotors in charge of the traction.

- Base1. This base contains all the robot's wiring and the battery supports, as well as the converters that allow communication between the camera and the netbook

- PC casing. It is mounted on top of Base1, and its main function is to protect the netbook from possible impacts.

- Manipulator support. It is fixed on Base1 and rests on the PC casing to provide more stability and strength to the manipulator.

- Microcontroller casing. Its function is only to protect the microcontroller and its servo controlling board from dust as well as from possible impacts, in addition to avoiding possible accidental disconnections of elements like the sensors, servomotors, artificial vision camera, and batteries. Figure 4 shows the above mentioned elements, assembled to form the frame.

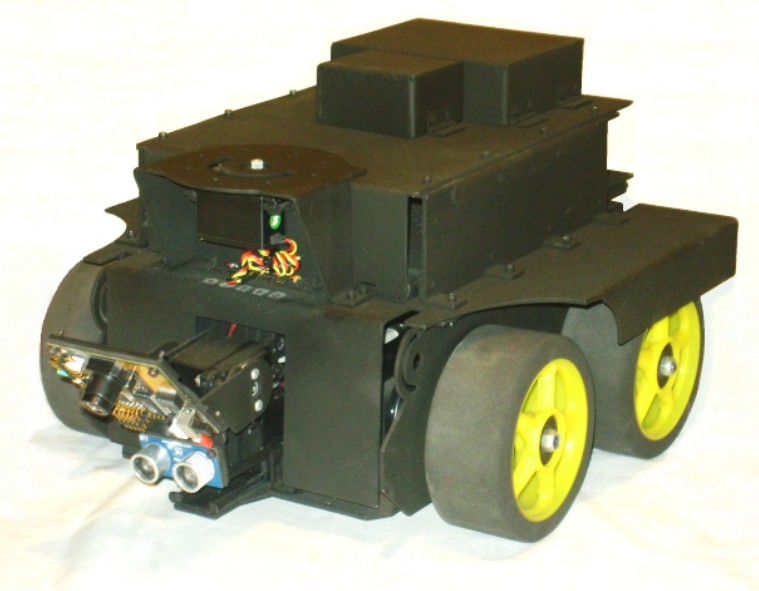

Fig. 4. Frame of the mobile robot.

\subsection{Manipulator}

This manipulator has four DOF on the same axle, all rotational, and a terminal effector composed of a clamp. Of these DOF, the first one allows a $180^{\circ}$ turning motion, to be able to handle and deposit the grasped objects; the second allows the reaching motion and practically has a function similar to that of the shoulder joint; the third allows bringing close, fulfilling the function of the elbow, also with a $180^{\circ}$ motion; and the fourth allows a fine and precise motion, fulfilling a function similar to the motion of the human wrist, and it also moves over $180^{\circ}$. The terminal effector allows grasping objects, and in general fulfills the function of the human fingers.

Figure 5 is a picture of the manipulator, composed of the following mechanical elements: base manipulator, arm, forearm, wrist, and terminal effector.

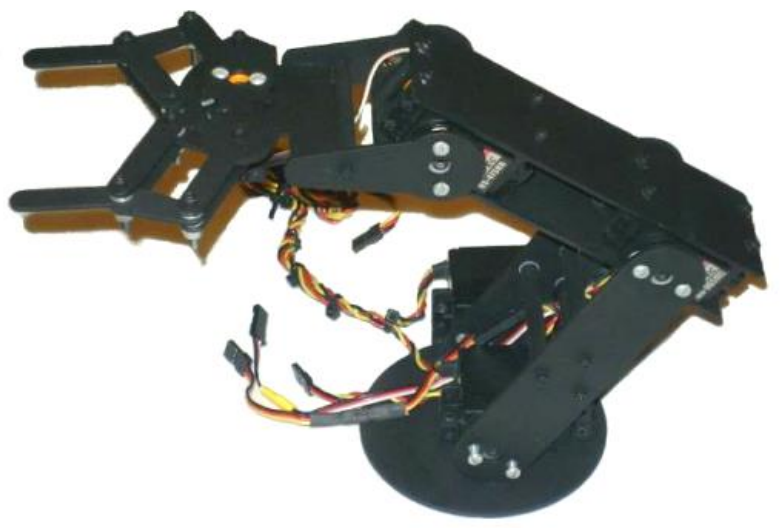

Fig. 5. Manipulator arm.

\subsection{Camera manipulator}

The camera manipulator consists of the following elements:

- Camera-Sensor support. It supports the camera as well as the ultrasonic sensor, and it also performs the PAN motion by means of the servomotor.

- TILT support. It is the part that supports the servomotor in charge of performing the PAN movement.

- Cart. It has the function of sliding the artificial vision camera into or out of the frame of the mobile robot, to expand its vision range. 
- Adjustment support. It consists of two parts that are fixed on base 0 , whose function is to provide mechanical adjustment to the cart.

These three mechanical parts, assembled, constitute the mobile robot shown in Figure 6.

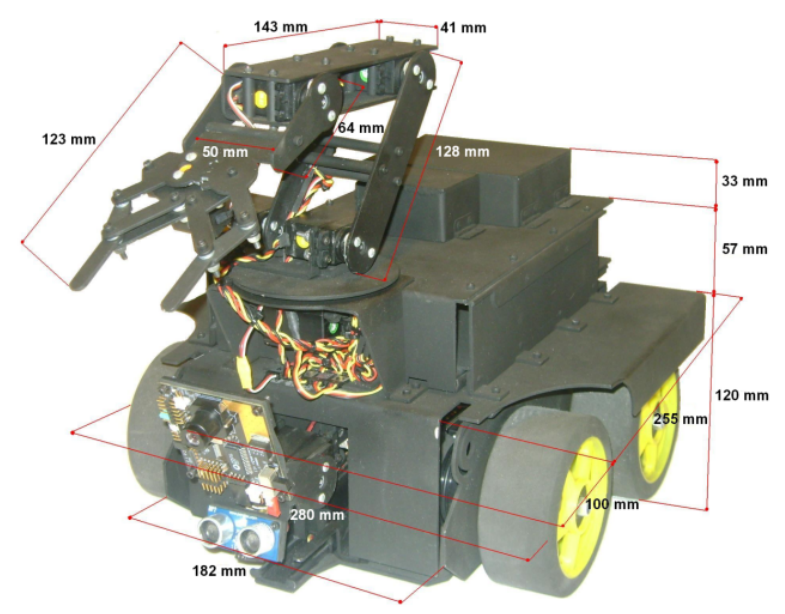

Fig. 6. Designed and implemented mobile robot.

\section{Programming and Results}

The microcontroller used for programming the mobile robot is a Basic Stamp, whose programming language is Basic. On the other hand, the commands of the artificial vision camera are very important for the development of object recognition, because if one of these commands is poorly configured, the recognition cannot take place. In addition to these commands, the artificial vision camera has others that give it different work characteristics and properties. The advantage of using this artificial vision camera is that, by means of its own commands, it allows the use of a technique for recognizing objects by means of their color. The mobile robot also has a netbook with the purpose of making it carry out specific tasks and work in general, as complex as its programming and its sensors allow.

\section{Conclusions}

The design, construction and programming of a mobile robot controlled by means of artificial vision, specifically by means of the recognition of different color objects, has been presented. This mobile robot also has a notebook that provides it with a large artificial intelligence capacity, which depends mostly on the programming it is given.

A mechanically robust robot has been built and a system has been designed that allows the mobility of two sensors jointly, i.e., artificial vision camera and distance sensor. This makes it possible to improve the range of artificial vision, over approximately $180^{\circ}$, achieving precise positioning of the mobile robot.

The Basic Stamp microcontroller provides efficiency in the algorithms, allowing good coordination between all the constitutive elements of the mobile robot.

The artificial vision camera, CMUCam 2, provides the mobile robot with great autonomy thanks to its excellent interaction with its surrounding world.

Having this mobile robot makes it possible to carry out interesting developments in various areas of mobile robotics.

\section{Acknowledgements}

This work was supported by the Mecesup USA1298 Project of the Universidad de Santiago de Chile, Chile.

\section{References}

1. L. Caracciolo, A. de Luca, S. Iannitti, Trajectory tracking control of a four wheel differentially driven mobile robot, in: IEEE Int. Conf. Robot. Autom., IEEE, 1999: pp. 2632-2638. doi:10.1109/ROBOT.1999.773994.

2. A. Ollero, Robótica: manipuladores y robots móviles, 1st ed., Marcombo, Barcelona, 2006.

3. R. Silva, J. García, V. Barrientos, M. Molina, V. Hernández, G. Silva, State of the art of the movable wheels robots, Telematique. 6 (2007).

4. D. Holz, M. Nieuwenhuisen, D. Droeschel, J. Stückler, A. Berner,
J. Li, et al., Active recognition and manipulation for mobile robot bin picking, in: F. Röhrbein, G. Veiga, C. Natale (Eds.), Gearing Up Accel. Cross-fertilization between Acad. Ind. Robot. Res. Eur., Springer International Publishing, Cham, 2014. doi:10.1007/978-3319-03838-4 7.

5. X. Wang, H. Zhu, D. Zhang, D. Zhou, X. Wang, Vision-based detection and tracking of a mobile ground target using a fixed-wing UAV, Int. J. Adv. Robot. Syst. (2014) 1. doi:10.5772/58989. 